\title{
Dynamical Bar Instability in Relativistic Rotating Stars
}

\author{
Motoyuki Saijo *, Masaru Shibata * ${ }^{*}$, \\ Thomas W. Baumgarte * and Stuart L. Shapiro *ł \\ * Department of Physics, University of Illinois at Urbana-Champaign, \\ 1110 West Green Street, Urbana, Illinois 61801-3080 \\ $\dagger$ Department of Earth Science and Astronomy, Graduate School of Arts and Science, \\ University of Tokyo, 3-8-1 Komaba, Meguro, Tokyo 153-8902, Japan \\ $¥$ Department of Astronomy and NCSA, University of Illinois at Urbana-Champaign, \\ 1002 West Green Street, Urbana, Illinois 61801
}

\begin{abstract}
We study by computational means the dynamical stability against barmode deformation of rapidly and differentially rotating stars in a post-Newtonian approximation of general relativity. We vary the compaction of the star $M / R$ (where $M$ is the gravitational mass and $R$ the equatorial circumferential radius) between 0.01 and 0.05 to isolate the influence of relativistic gravitation on the instability. For compactions in this moderate range, the critical value of $\beta=T / W$ for the onset of the dynamical instability (where $T$ is the rotational kinetic energy and $\mathrm{W}$ the gravitational binding energy) slightly decreases from $\sim 0.26$ to $\sim 0.25$ with increasing compaction for our choice of the differential rotational law. Combined with our earlier findings based on simulations in full general relativity for stars with higher compaction, we conclude that relativistic gravitation enhances the dynamical bar-mode instability, i.e. the onset of instability sets in for smaller values of $\beta$ in relativistic gravity than in Newtonian gravity. We also find that once a triaxial structure forms after the bar-mode perturbation saturates in dynamically unstable stars, the triaxial shape is maintained, at least for several rotational periods. To check the reliability of our numerical integrations, we verify that the general relativistic Kelvin-Helmholtz circulation is well-conserved, in addition to rest-mass and total mass-energy, linear and angular momentum. Conservation of circulation indicates that our code is not seriously affected by numerical viscosity. We determine the amplitude and frequency of the quasi-periodic gravitational waves emitted during the bar formation process using the quadrupole formula.
\end{abstract}

Stars in nature are usually rotating and subject to nonaxisymmetric rotational instabilities. An exact treatment of these instabilities exists only for incompressible equilibrium fluids in Newtonian gravity. For these configurations, global rotational instabilities arise from non-radial toroidal modes when $\beta \equiv T / W$ exceeds a certain critical value. Here $T$ and $W$ are the rotational kinetic and gravitational binding energies. There exist two different mechanisms and corresponding timescales 
for bar-mode instabilities. Uniformly rotating, incompressible stars in Newtonian theory are secularly unstable to bar-mode formation when $\beta \geq \beta_{\mathrm{sec}} \simeq 0.14$. This instability can grow only in the presence of some dissipative mechanism, like viscosity or gravitational radiation. The growth time is determined by the dissipative timescale, which is usually much longer than the dynamical timescale of the system. By contrast, a dynamical instability to bar-mode formation occurs when $\beta \geq \beta_{\text {dyn }} \simeq 0.27$. This instability is independent of any dissipative mechanisms, and the growth time is the hydrodynamic timescale of the system.

Determining the onset of the dynamical bar-mode instability, as well as the subsequent evolution of an unstable star, requires a fully nonlinear hydrodynamic simulation. Recently, simulations in Newtonian theory $[1,2]$ have shown that a higher degree of differential rotation enhances the onset of dynamical instability. Simulations in full general relativity [3] suggest that nonlinear gravitation has a similar effect.

The purpose of post-Newtonian simulations [4] is twofold. We verify that relativistic gravitation alone enhances the dynamical instability, i.e. $\beta_{\text {dyn }}$ decreases with increasing compaction. We also show that in unstable configurations, the bar persists for at least several rotational periods $[5,6]$ so that unstable stars are promising sources of quasi-periodic gravitational waves.
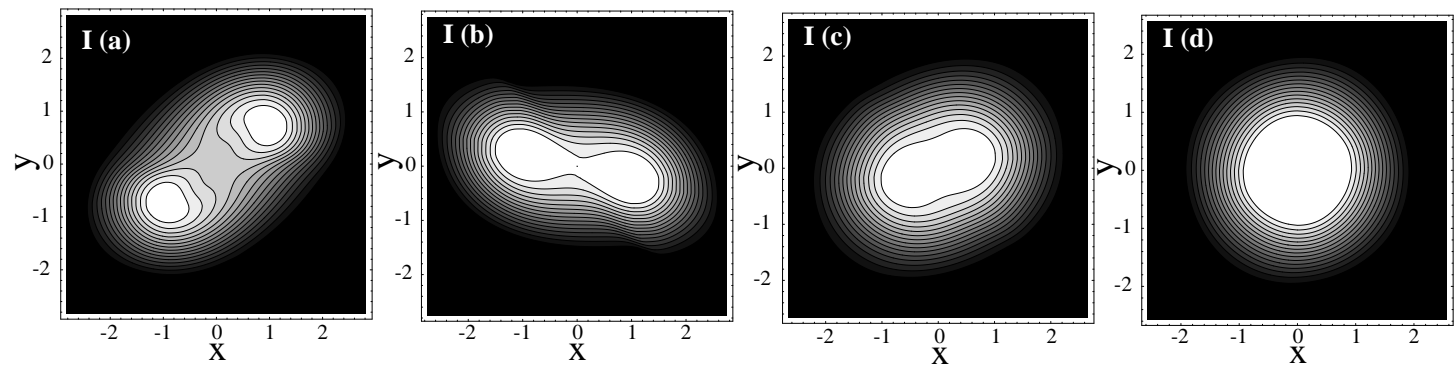

FIGURE 1. Final density contours in the equatorial plane for differentially rotating stars all of compaction $M / R=0.05$ and varying values of $\beta$. The contour lines denote densities $\rho_{*}=1.3 i \times 10^{-3}(i=1, \ldots, 15)$. Snapshots are plotted at the following times and initial $\beta$ : (a) $t / P_{c}=2.72, \beta=0.265$ (b) $t / P_{c}=3.66, \beta=0.259$, (c) $t / P_{c}=7.77, \beta=0.249$, and (d) $t / P_{c}=8.16, \beta=0.238$. Here, $P_{c}$ is the central rotation period.

We perform post-Newtonian simulations of rapidly and differentially rotating stars to investigate general relativistic effects on the dynamical bar-mode instability for small compactions $M / R \leq 0.05$. As a criterion for stability, we checked whether the distortion parameter follows an exponential growth. The formation of a bar is also apparent in the snapshots of density contours (See Fig. 1 for $M / R=0.05$; Figs. $4-7$ of Ref. [4]). These plots clearly exhibit a triaxial structure for the unstable models, while for stable models the density distribution hardly changes during the evolution. By combining these post-Newtonian results with the fully relativistic simulations [3] for configurations of higher compaction, we conclude that the critical value of $\beta=\beta_{\text {dyn }}$ decreases with increasing $M / R$ (Fig. 2). Thus, 


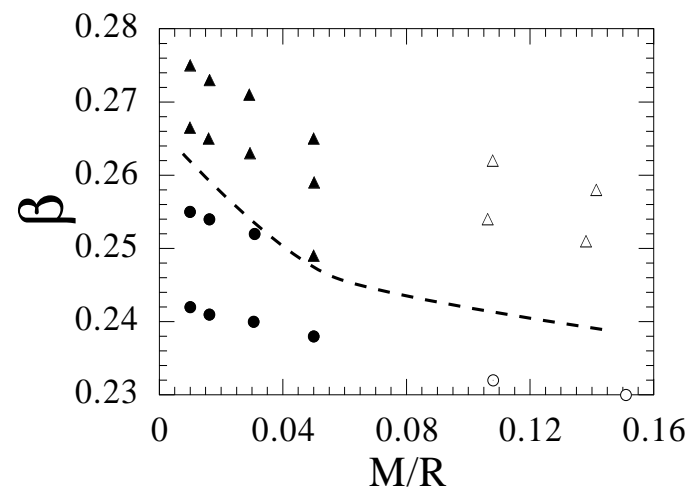

FIGURE 2. Summary of our dynamical stability analysis. All our models are plotted in a $\beta$ versus $M / R$ plane, with stable stars denoted by circles and unstable stars by triangles. The solid circles and triangles are the models studied in our post-Newtonian simulations [4]; the open circles and triangles are the models explored in full general relativity [3]. We conclude that the critical value of $\beta=\beta_{\mathrm{dyn}}$ slightly decreases with increasing compaction $M / R$. This trend is emphasized by the dotted line, which approximates $\beta_{\mathrm{dyn}}$ as a function of $M / R$.

relativistic gravitation enhances the bar-mode instability.

We also confirm that bar-structure persists at least several rotation period by checking the conservation of circulation (Fig. 8 and Table 3 of Ref. [4]). In the presence of significant numerical viscosity, long-time evolution calculations become very unreliable and may lead, for example, to erroneous evolution of a saturated bar. We have shown that in our calculations the circulation is well conserved, implying that our code is at most very weakly affected by numerical viscosity. We present a method for computing the circulation which can be applied in Newtonian, post-Newtonian and fully relativistic calculations.

Finally, we have calculated approximate gravitational waveforms in the quadrupole approximation, neglecting all post-Newtonian corrections (Fig. 10 of Ref. [4]). We found that for unstable stars a quasi-periodic oscillation with growing amplitude arises during the early bar formation. The bar-structure persists for several rotation periods, implying that bar-unstable stars are promising sources of quasi-periodic gravitational waves.

\section{REFERENCES}

1. Tohline, J. E. and Hachisu, I., Astrophys. J. 361, 394 (1990).

2. Pickett, B. K., Durisen, R. H., and Davis, G. A., Astrophys. J. 458, 714 (1996).

3. Shibata, M., Baumgarte, T. W., and Shapiro, S. L., Astrophys. J. 542, 453 (2000).

4. Saijo, M., Shibata, M., Baumgarte, T. W., and Shapiro, S. L., Astrophys. J. 548, 919 (2001).

5. New, K. C. B., Centrella, J. M., and Tohline, J. E., Phys. Rev. D 62, 064019 (2000).

6. Brown, J. D., Phys. Rev. D 62, 084024 (2000). 